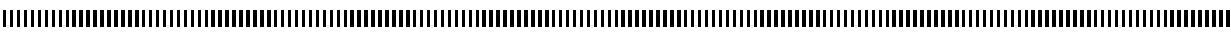

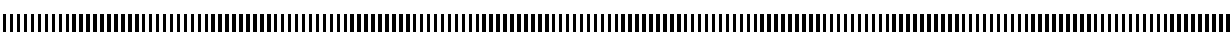
|

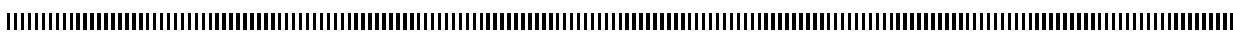
| |

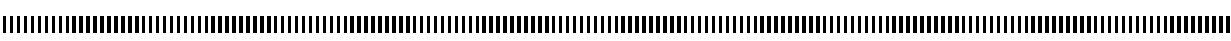

\title{
Approche structurelle des systèmes, de la géométrie à la théorie des graphes
}

\author{
Jean-Michel Dion ${ }^{\mathrm{a}}$ - Christian Commaulta, ${ }^{\mathrm{*}}$ \\ GIPSA-Lab Grenoble, Département Automatique , \\ ENSIEG-BP 46, 38402 Saint Martin d'Hères Cedex, France \\ Jean-Michel.Dion@inpg.fr \\ * Corresponding author \\ Christian.Commault@inpg.fr
}

IIIIIIIIIIIIIIIIIIIIIIIIIIIIIIIIIIIIIIIIIIIIIIIIIIIIIIIIIIIIIIIIIIIIIIIIIIIIIIIIIIIIIIIIIIIIIIIIIIIIIIIIIIIIIIIIIIIIIIIIIIIIIIIIIIIIIIIIIIIIIIIIIIIIII

RÉSUMÉ. Dans ce travail présenté au colloque scientifique organisé en l'honneur de Claude Lobry nous nous focalisons sur une approche structurelle des systèmes qui a été le fil conducteur de beaucoup de nos travaux. Les capacités de modélisation de cette approche et la puissance de l'outil graphique associé sont mises en lumière. A titre d'illustration on considère le problème de rejet de perturbations par retour de mesure en utilisant des outils géométriques et graphiques.

ABSTRACT. In this work, which was presented at the conference in honor of Claude Lobry, we focus on a structural approach of systems which was the mainstream of our research. The modeling ability of this approach and the power of the associated graph tools are enlightened. As an illustration we consider the disturbance decoupling problem by measurement feedback and solve this problem using geometric and graph techniques

MOTS-CLÉS : Systèmes structurés, Approche géométrique, Graphes, Rejet de perturbations

KEYWORDS : Structured systems, Geometric approach, Graphs, Disturbance decoupling

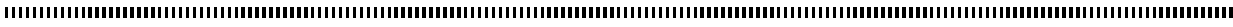




\section{Introduction}

Cette présentation a pour objectif de résumer quelques propriétés structurelles des systèmes dynamiques, en se focalisant sur une classe de systèmes que nous avons particulièrement étudiée, celle des systèmes structurés. Cette classe de systèmes linéaires paramétrés est bien adaptée à la modélisation des systèmes physiques. On verra que des propriétés génériques de ces systèmes peuvent être déduites simplement de l'analyse d'un graphe représentant le système considéré.

L'approche structurelle a été initiée par Lin dans les années 70 pour étudier l'atteignabilité générique [1]. De nombreux auteurs se sont intéressés à ce type de modèle structuré et on trouvera des références dans les livres [2,3,4] et dans le papier de synthèse [5].

On présentera tout d'abord les propriétés de base des systèmes structurés et des graphes associés. On se focalisera sur le problème du rejet de perturbations par retour de mesure. En mélangeant les approches géométriques et graphiques on donne des condition génériques de solubilité de ce problème. Les résultats et notions sont illustrés sur un exemple pédagogique.

\section{Systèmes structurés}

\subsection{Modélisation structurée des systèmes dynamiques}

En pratique nous sommes souvent confrontés à la situation suivante lors de la modélisation d'un système physique : le système peut contenir des paramètres fixes qui représentent le rôle particulier joué par certaines variables dans le système. Ceci peut arriver si le système est composé, par exemple, de sous-ensembles connectés en série. Une autre raison pour l'existence de paramètres fixes est l'existence de relations algébriques entre variables, par exemple lorsqu'une variable est la dérivée d'une autre. Enfin l'absence de relation directe entre variables se traduit par un paramètre nul dans le modèle.

Le modèle peut contenir également des paramètres qui représentent des relations empiriques ou des lois de la physique qui lient les variables entre elles. De tels paramètres sont par exemple des masses, des inerties, ..., obtenues par identification. Une caractéristique commune de ces paramètres est qu'ils sont sujets aux erreurs de modélisation. Une autre situation commune est celle de la linéarisation des modèles, dans ce cas, la structure zéro/non zéro est fixe mais la valeur des paramètres non nuls varie avec le point de fonctionnement. Comme illustré Figure 1, le modèle linéarisé d'une colonne de distillation montre une structure invariante par rapport au point de fonctionnement [6]

L'approche habituelle des systèmes linéaires souffre de plusieurs inconvénients par rapport aux remarques précédentes. D'abord elle ne permet pas de prendre en compte l'information structurelle sur les paramètres, ensuite elle suppose ces paramètres parfaitement connus. Bien sûr un certain nombre d'approches permettent de prendre en compte l'aspect incertain du système, par exemple les modèles stochastiques ou les études de robustesse. Ces approches permettent par optimisation l'obtention de lois de commande stabilisantes mais ne permettent pas d'analyser la structure interne du système. Elles ne conduisent en général qu'à des conditions suffisantes. Une notion intéressante qui permet de prendre en compte quelques unes des observations précédentes est la notion de système 
structuré dans une représentation d'état avec coefficients paramétrés [5]. La structure est essentiellement déterminée par la position des zéros fixes dans les matrices de la représentation d'état. A un système structuré on peut associer de façon naturelle un graphe orienté. Les propriétés génériques du système peuvent alors souvent être caractérisées très simplement en termes de propriétés du graphe associé. Ceci rend très intuitifs certains résultats. Cette modélisation a les caractéristiques suivantes :

1) elle permet de prendre en compte une partie importante de l'information structurelle provenant des lois physiques et de la décomposition du système en sous-systèmes,

2) elle donne à travers le graphe une représentation visuelle de cette structure,

3) elle permet l'étude des propriétés du système presque indépendamment des valeurs des paramètres inconnus, ces paramètres inconnus étant en général des fonctions des paramètres physiques,

4) le coût de calcul pour tester une propriété est en général très réduit, ce qui permet de traiter des problèmes de grande dimension, en particulier s'ils sont très "creux".

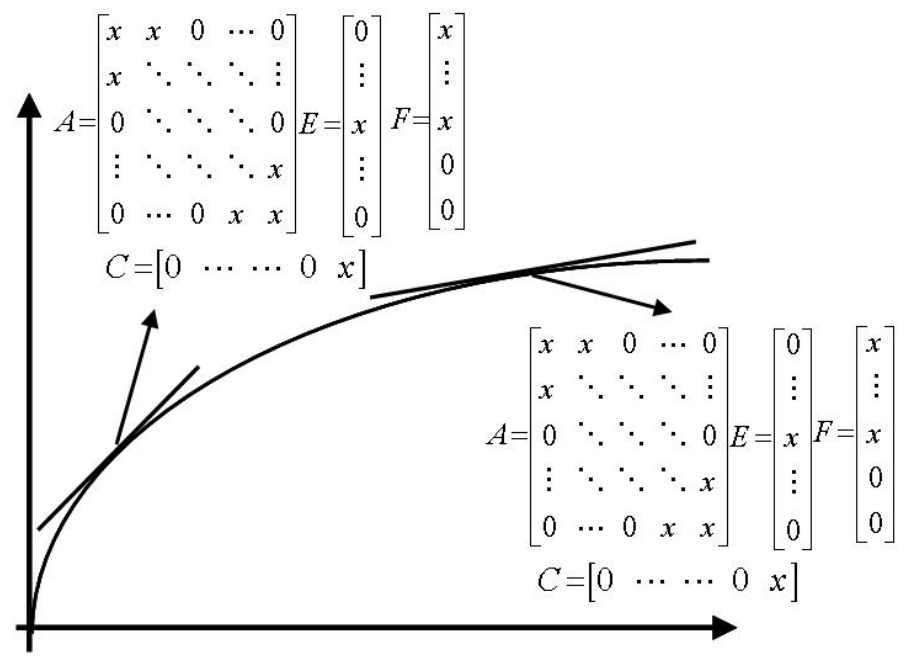

Figure 1. Le modèle d'une colonne de distillation

\subsection{Le modèle}

On considère une classe de systèmes linéaires dépendant de paramètres (les systèmes linéaires structurés) décrite par le modèle suivant :

$$
\Sigma_{\lambda}:\left\{\begin{array}{l}
\dot{x}(t)=A x(t)+B u(t) \\
y(t)=C x(t)
\end{array}\right.
$$

où $x(t) \in \mathbb{R}^{n}$ est le vecteur d'état, $u(t) \in \mathbb{R}^{m}$ est le vecteur d'entrée et $y(t) \in \mathbb{R}^{p}$ est le vecteur des sorties mesurées. $A, B, C$ sont des matrices de dimensions appropriées telles que les éléments de la matrice composite $J=\left[\begin{array}{cc}A & B \\ C & 0\end{array}\right]$ sont soit des zeros fixes soit des paramètres indépendants (non reliés par des relations algébriques). $\lambda=\left\{\lambda_{1}, \lambda_{2}, \ldots, \lambda_{k}\right\}$ 
contient l'ensemble des paramètres indépendants de la matrice $J$. Sur $\Sigma_{\lambda}$ on étudiera des propriétés génériques (vraies pour presque toute valeur des paramètres). Dans cette présentation on utilisera à la fois des matrices structurées (dépendant de paramètres) et des matrices à éléments réels connus, par simplicité de notation on omettra la dépendance en $\lambda$ de ces matrices, le contexte évitera toute ambiguïté.

A un tel système on peut facilement associer un graphe $G\left(\Sigma_{\lambda}\right)=(V, W)$ dont l'ensemble des sommets est $V=U \cup X \cup Y$ où $U, X$ et $Y$ sont les entrées, états et sorties donnés respectivement par $\left\{u_{1}, u_{2}, \ldots, u_{m}\right\},\left\{x_{1}, x_{2}, \ldots, x_{n}\right\}$ et $\left\{y_{1}, y_{2}, \ldots, y_{p}\right\}$. L'ensemble d'arcs est $W=\left\{\left(u_{i}, x_{j}\right) \mid B_{j i} \neq 0\right\} \cup\left\{\left(x_{i}, x_{j}\right) \mid A_{j i} \neq 0\right\} \cup\left\{\left(x_{i}, y_{j}\right) \mid C_{j i} \neq 0\right\}$, où $A_{j i}$ (resp. $\left.B_{j i}, C_{j i}\right)$ est l'élément $(j, i)$ de la matrice $A$ (resp. $B, C$ ).

De plus rappelons qu'un chemin dans $G\left(\Sigma_{\lambda}\right)$ reliant le sommet $i_{0}$ au sommet $i_{l}$ est une suite d'arcs $\left(i_{0}, i_{1}\right),\left(i_{1}, i_{2}\right), \ldots,\left(i_{l-2}, i_{l-1}\right),\left(i_{l-1}, i_{l}\right)$ telle que $i_{t} \in V$ pour $t=$ $0,1, \ldots, l$ et $\left(i_{t-1}, i_{t}\right) \in W$ pour $t=1,2, \ldots, l$.

Un ensemble de chemins sans sommet commun est dit sommet-disjoint. Un couplage $V_{1}-V_{2}$ de dimension $k$, où $V_{1}$ et $V_{2}$ sont inclus dans $V$, est un ensemble de $k$ chemins sommet-disjoints entre $V_{1}$ et $V_{2}$. Un tel couplage est maximal quand $k$ est maximal.

De nombreux résultats ont été obtenus pour les systèmes structurés en utilisant le graphe associé. Par exemple on peut caractériser l'observabilité comme suit [1,4].

Proposition 1 Soit $\Sigma_{\lambda}$ le système structuré défini par (1) et son graphe associé $G\left(\Sigma_{\lambda}\right)$. Le système (en fait la paire $(C, A)$ ) est structurellement observable si et seulement si :

- tous les sommets d'état sont reliés à un sommet de sortie par un chemin,

- il existe un ensemble de circuits et de chemins état-sortie sommets-disjoints qui couvre tous les sommets d'état.

On a également le résultat suivant qui exprime simplement le rang générique de la matrice de transfert entre les entrées et les sorties du système [7].

Proposition 2 Soit $\Sigma_{\lambda}$ le système structuré défini par (1) et son graphe associé $G\left(\Sigma_{\lambda}\right)$. Le rang générique de $C(s I-A)^{-1} B$ est égal à la dimension maximale d'un couplage entrée-sortie dans $G\left(\Sigma_{\lambda}\right)$.

Exemple 1 Nous allons maintenant illustrer les notions et résultats précédents sur un exemple. Considérons un système structuré $\Sigma_{\lambda}$ de type (1) avec 3 états, 3 entrées et 2 sorties :

$$
\begin{gathered}
A=\left[\begin{array}{ccc}
0 & 0 & 0 \\
\lambda_{1} & 0 & 0 \\
0 & \lambda_{2} & 0
\end{array}\right], B=\left[\begin{array}{ccc}
\lambda_{3} & 0 & 0 \\
0 & \lambda_{4} & 0 \\
0 & 0 & \lambda_{5}
\end{array}\right] \\
C=\left[\begin{array}{ccc}
0 & \lambda_{6} & 0 \\
0 & 0 & \lambda_{7}
\end{array}\right]
\end{gathered}
$$

Les éléments non nuls des matrices sont les paramètres libres $\lambda=\left(\lambda_{1}, \lambda_{2}, \ldots, \lambda_{7}\right)$. Le graphe associé $G\left(\Sigma_{\lambda}\right)$ est donné Figure 2.

Ce système est structurellement observable par la Proposition 1. En fait il existe un 


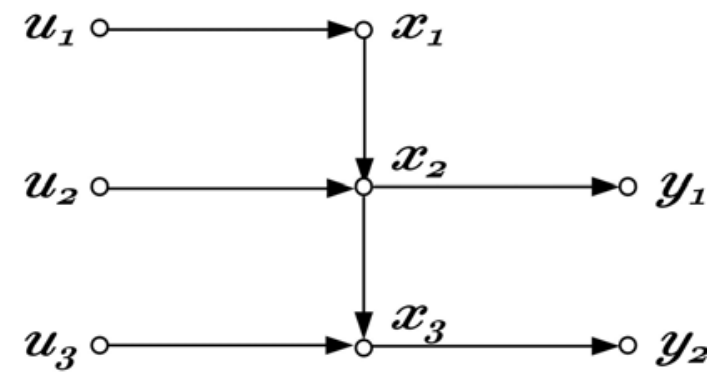

Figure 2. Graphe $G\left(\Sigma_{\lambda}\right)$ de l'exemple 1

chemin entre chaque état et une sortie et un ensemble de chemins état-sortie sommetsdisjoints $\left(x_{1}, x_{2}, y_{1}\right)$ et $\left(x_{3}, y_{2}\right)$ qui couvre tous les sommets d'état. On peut vérifier que la matrice de transfert du système

$$
T(s)=\left[\begin{array}{ccc}
\lambda_{1} \lambda_{3} \lambda_{6} s^{-2} & \lambda_{4} \lambda_{6} s^{-1} & 0 \\
\lambda_{1} \lambda_{2} \lambda_{3} \lambda_{7} s^{-3} & \lambda_{2} \lambda_{4} \lambda_{7} s^{-2} & \lambda_{5} \lambda_{7} s^{-1}
\end{array}\right]
$$

est bien de rang générique 2 car il existe un couplage de dimension 2 dans le graphe.

\subsection{Le rejet de perturbations, approche géométrique}

Le problème du rejet de perturbations est un problème très classique qui a reçu une solution élégante en termes géométriques dans les années 70 [8,9]. Le problème peut se formuler de la façon suivante, on considère le système :

$$
\Sigma_{q}:\left\{\begin{array}{l}
\dot{x}(t)=A x(t)+B u(t)+E q(t) \\
y(t)=C x(t)
\end{array}\right.
$$

où $q(t) \in \mathbb{R}^{d}$ est une entrée additionnelle appelée perturbation dont on aimerait qu'elle n'influence pas la sortie. On cherche une commande par retour d'état, $u=F x$, telle que la matrice de transfert perturbation-sortie soit nulle, c'est-à-dire :

$$
T_{F}(s)=C(s I-A-B F)^{-1} E=0 .
$$

Une condition nécessaire et suffisante bien connue pour que ce problème ait une solution est que :

$$
\mathcal{E} \subset \mathcal{V}^{*}
$$

où $\mathcal{E}$ est l'image de $E$ et $\mathcal{V}^{*}$ est le plus grand sous-espace $(A, B)$-invariant de $\operatorname{Ker} C$. Une introduction simple à la théorie géométrique est donnée dans [10]. En fait le sousespace $\mathcal{V}^{*}$ se calcule facilement de façon itérative, il s'agit du plus grand sous-espace non observable qu'on peut obtenir par retour d'état.

Quand la perturbation est accessible à la mesure, la loi de commande s'écrit $u=F x+J q$ et on cherche alors $F$ et $J$ telles que la matrice de transfert en boucle fermée soit nulle :

$$
T_{F, J}(s)=C(s I-A-B F)^{-1}(B J+E)=0 .
$$

La condition de solubilité du problème devient :

$$
\mathcal{E} \subset \mathcal{V}^{*}+\mathcal{B}
$$


où $\mathcal{B}$ représente l'Image de $B$.

\subsection{Le rejet de perturbations, approche graphique}

Dans le contexte des systèmes structurés, le système $\Sigma_{q \lambda}$ s'écrit :

$$
\Sigma_{q \lambda}:\left\{\begin{array}{l}
\dot{x}(t)=A x(t)+B u(t)+E q(t) \\
y(t)=C x(t)
\end{array}\right.
$$

et on peut lui associer le graphe $G\left(\Sigma_{q \lambda}\right)$ en ajoutant à $G\left(\Sigma_{\lambda}\right)$ les sommets et les arcs correspondant aux perturbations. Pour des raisons de simplicité on considère d'abord, dans ce cas, le problème du rejet de perturbation avec mesure, $u=F x+J q$, on a alors [11].

Théorème 1 Considérons un système structuré du type (7) dont le graphe est $G\left(\Sigma_{q \lambda}\right)$. Le problème du rejet de perturbation par retour d'état avec mesure de la perturbation est génériquement soluble si et seulement si

1) Un couplage maximum de $U$ à $Y$ dans $G\left(\Sigma_{\lambda}\right)$ et un couplage maximum de $U \cup Q$ à $Y$ dans $G\left(\Sigma_{q \lambda}\right)$ ont la même taille notée $r$.

2) $L_{r}=L_{d, r}$, où $L_{r}$ est le nombre minimum de sommets de $X$ contenus dans un couplage de taille $r$ de $U$ à $Y$ dans $G\left(\Sigma_{\lambda}\right)$ et $L_{d, r}$ est le nombre minimum de sommets de $X$ contenus dans un couplage de taille $r$ de $U \cup Q$ à $Y$ dans $G\left(\Sigma_{q \lambda}\right)$.

Ce théorème exprime le fait que pour résoudre le problème les commandes doivent être suffisamment nombreuses et rapides pour éliminer l'influence des perturbations avant qu'elles n'atteignent la sortie. Ceci peut être illustré par les exemples simples suivants.

Exemple 2 Considérons d'abord le système dont le graphe est visualisé Figure 3.

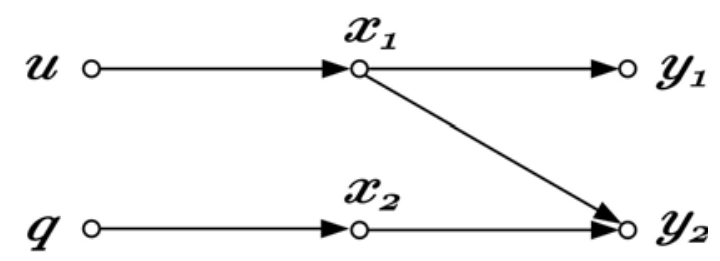

Figure 3. Graphe $G\left(\Sigma_{\lambda}\right)$ de l'exemple 2

Il est clair qu'un couplage maximum entre $U$ et $Y$ est de taille 1 alors que'un couplage maximum entre $U \cup Q$ et $Y$ est de taille 2. La condition 1 du Théorème 1 n'est pas satisfaite, le problème de rejet de perturbation avec mesure de la perturbation n'a donc pas de solution. En effet, la commande ne peut pas éliminer l'effet de la perturbation sur $y_{2}$ sans influencer $y_{1}$.

Exemple 3 Considérons maintenant le système dont le graphe est visualisé Figure 4.

La condition 1 est clairement vérifiée sur le graphe avec un couplage de taille 1. La seconde condition est également satisfaite puisque $L_{1}=L_{d, 1}=1$ donc, le problème de 


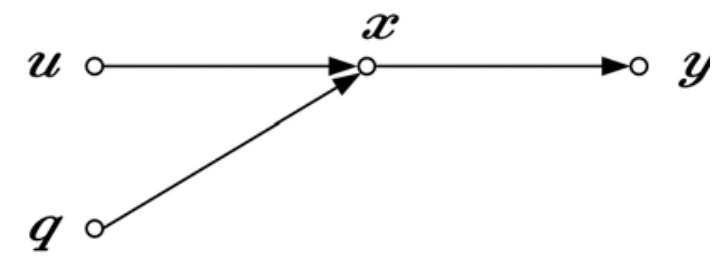

Figure 4. Graphe $G\left(\Sigma_{\lambda}\right)$ de l'exemple 3

rejet de perturbation avec mesure de la perturbation a une solution. La perturbation étant connue on peut calculer une commande qui annule son effet en $x$.

Dans le cas où la perturbation n'est pas accessible à le mesure, alors $J=0$, nous avons le résultat suivant.

Théorème 2 Considérons un système structuré du type (7) dont le graphe est $G\left(\Sigma_{q \lambda}\right)$. Le problème du rejet de perturbation par retour d'état sans mesure de la perturbation est génériquement soluble si et seulement si

1) Un couplage maximum de $U$ à $Y$ dans $G\left(\Sigma_{\lambda}\right)$ et un couplage maximum de $U \cup Q$ à $Y$ dans $G\left(\Sigma_{q \lambda}\right)$ ont la même taille notée $r$.

2) $L_{r}=L_{d, r}^{\prime}$, où $L_{r}$ est le nombre minimum de sommets de $X$ contenus dans un couplage de taille $r$ de $U$ à $Y$ dans $G\left(\Sigma_{\lambda}\right)$ et $L_{d, r}^{\prime}$ est le nombre minimum de sommets de $X \cup U$ contenus dans un couplage de taille $r$ de $U \cup Q$ à $Y$ dans $G\left(\Sigma_{q \lambda}\right)$.

Dans le cas où la perturbation n'est pas mesurable, l'information sur cette perturbation provient de la mesure de l'état, ce qui fait perdre du temps (un pas d'échantillonnage pour les systèmes discrets). La condition du Théorème 2 est semblable à celle du Théorème 1 modulo ce retard de un pas. Si on reprend l'exemple ci-dessus, la condition 2 du Théorème 2 n'est pas satisfaite. En effet, sans mesure de la perturbation, lorsque l'effet de celle-ci sera mesuré en $x$ il sera trop tard pour agir.

Dans le contexte des systèmes structurés le sous-espace $(A, B)$-invariant maximum contenu dans $\operatorname{Ker} C$ dépend alors des paramètres, on le note $\mathcal{V}_{\lambda}^{*}$, ce qui signifie qu'il varie dans $\mathbb{R}^{n}$ quand $\lambda$ varie dans $\mathbb{R}^{k}$, mais sa dimension reste génériquement constante. On peut définir un sous-espace, appelé partie fixe de $\mathcal{V}_{\lambda}^{*}$ et noté $\mathcal{V}^{*}{ }_{F}$, qui est le plus grand sousespace fixe contenu dans presque tous les $\mathcal{V}_{\lambda}^{*}$ quand $\lambda$ varie dans $\mathbb{R}^{k}$, voir Figure 5. Le sous-espace $\mathcal{V}^{*}{ }_{F}$ peut être caractérisé simplement sur le graphe de la manière suivante $\mathcal{V}^{*}{ }_{F}=\left\{e_{i}\right.$ pour i tel que $\left.x_{i} \in \mathcal{I}^{*}\right\}$ [12] où $e_{i}$ est le $i$ ème vecteur de la base canonique de $\mathbb{R}^{n}$ et :

$\mathcal{I}^{*}=\left\{x_{i}, i \in\{1,2, \ldots, n\} \mid\right.$ la taille maximale d'un couplage de $U \cup x_{i} \grave{a}$

$Y$ dans $G\left(\Sigma_{q \lambda}\right)$ est la même que la taille maximale d'un couplage de

$U$ à $Y$ dans $G\left(\Sigma_{q \lambda}\right)$ et le nombre minimal de sommets de $X \cup U$ dans

les deux couplages est le même

$\mathcal{I}^{*}$ caractérise l'ensemble des $e_{i}$ tels que, si une perturbation arrive sur $e_{i}$ elle peut être rejetée par un retour d'état sans mesure de la perturbation.

Si on note $\mathcal{E}_{F}$ le plus petit sous-espace fixe contenant les $\mathcal{E}_{\lambda}$ quand $\lambda$ varie dans $\mathbb{R}^{k}$, on a l'équivalent en structurel du résultat de la condition (4) [13] : 


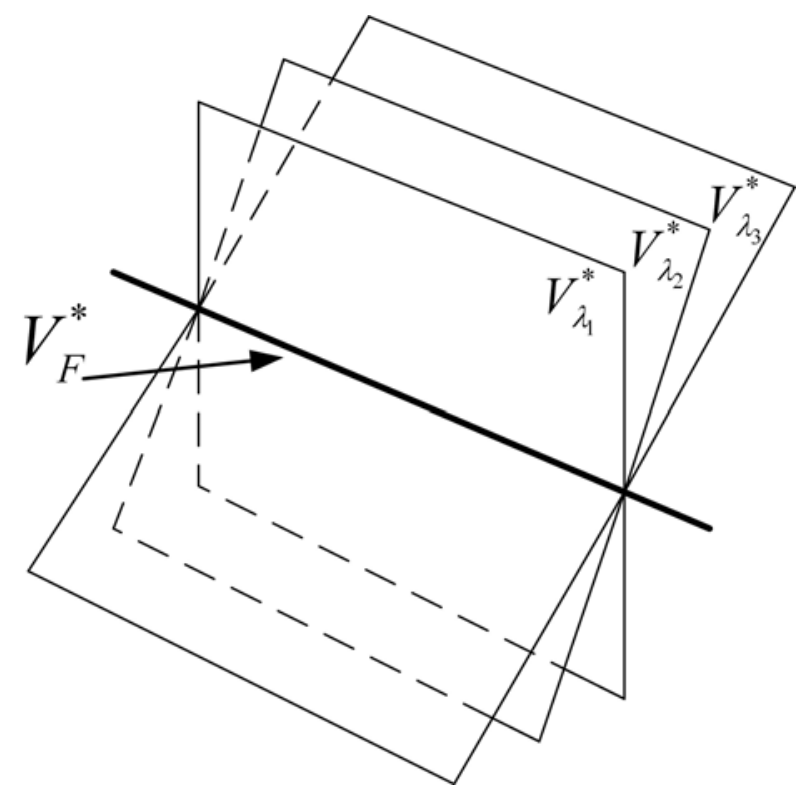

Figure 5. $\mathcal{V}^{*}{ }_{F}$ et la variation de $\mathcal{V}_{\lambda}^{*}$

Théorème 3 Considérons un système structuré du type (7) dont le graphe est $G\left(\Sigma_{q \lambda}\right)$. Le problème du rejet de perturbation par retour d'état sans mesure de la perturbation est génériquement soluble si et seulement si

$$
\mathcal{E}_{F} \subset \mathcal{V}_{F}^{*}
$$

\subsection{Le rejet de perturbations par retour de mesure}

Repartons du système (2) et supposons que les perturbations ne sont pas mesurables et que l'état n'est pas complètement accessible. On a un vecteur de sorties mesurées $z=$ $H x$. La loi de commande considérée est très générale, il s'agit d'un retour dynamique de mesure. Nous considérons donc le système suivant $\Sigma_{q z}$, voir Figure 6

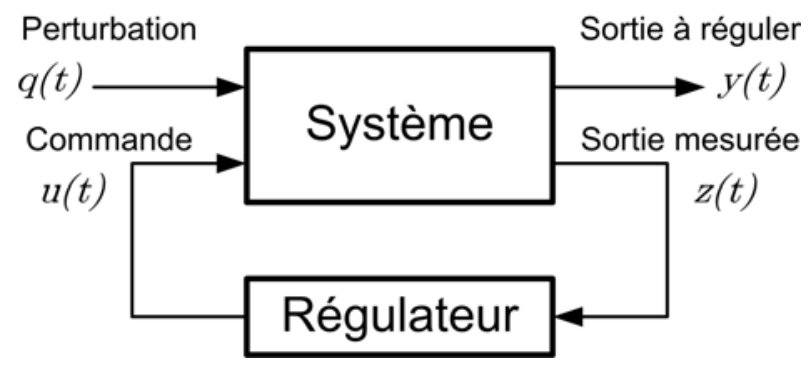

Figure 6. Le retour de mesure 


$$
\Sigma_{q z}:\left\{\begin{array}{l}
\dot{x}(t)=A x(t)+B u(t)+E q(t) \\
y(t)=C x(t) \\
z(t)=H x(t)
\end{array}\right.
$$

où $z(t) \in \mathbb{R}^{l}$. Le problème de rejet de perturbation revient à trouver un retour dynamique de mesure défini par

$$
\Sigma_{f b}:\left\{\begin{array}{l}
\dot{w}(t)=L w(t)+M z(t) \\
u(t)=N w(t)+P z(t)
\end{array}\right.
$$

où $w(t) \in \mathbb{R}^{\mu}$, tel que le transfert en boucle fermée entre $q$ et $y$ soit nul. En termes de matrices de transfert on a :

$$
\left[\begin{array}{l}
y(s) \\
z(s)
\end{array}\right]=\left[\begin{array}{ll}
G(s) & K(s) \\
M(s) & N(s)
\end{array}\right]\left[\begin{array}{l}
u(s) \\
q(s)
\end{array}\right]
$$

$u=F(s) y$ et $F(s)$ tel que

$$
G(s) F(s)(I-M(s) F(s))^{-1} N(s)+K(s)=0
$$

Ce problème a une solution très élégante en termes géométriques (voir $[14,15])$. Une condition nécessaire et suffisante pour que le problème ait une solution est que

$$
\mathcal{N}^{*} \subset \mathcal{V}^{*}
$$

où $\mathcal{N}^{*}$ est le plus petit sous-espace $(H, A)$-invariant contenant $\operatorname{Im} E$ et $\mathcal{V}^{*}$ le plus grand sous-espace $(A, B)$-invariant contenu dans $\operatorname{Ker} C$.

Quand le système est structuré le modèle devient

$$
\Sigma_{q z \lambda}:\left\{\begin{array}{l}
\dot{x}(t)=A x(t)+B u(t)+E q(t) \\
y(t)=C x(t) \\
z(t)=H x(t)
\end{array}\right.
$$

et le graphe associé est noté $G\left(\Sigma_{q z \lambda}\right)$. Le problème de rejet de perturbation revient alors à trouver un retour dynamique de mesure, qui dépendra des paramètres, tel que le transfert en boucle fermée entre $q$ et $y$ soit nul. Comme vu précédemment, $\mathcal{V}_{\lambda}^{*}$ varie en fonction des paramètres et sa partie fixe $\mathcal{V}_{F}^{*}$ correspond sur le graphe à $\mathcal{I}^{*}$ qui caractérise les perturbations qui peuvent être rejetées par retour d'état.

De manière duale, on définit l'ensemble $\mathcal{J}^{*}$.

$$
\begin{aligned}
& \mathcal{J}^{*}=\left\{x_{j}, j \in\{1,2, \ldots, n\} \mid \text { la taille maximale d'un couplage de } Q \grave{a}\right. \\
& Z \cup x_{j} \text { dans } G\left(\Sigma_{q z \lambda}\right) \text { est la même que la taille maximale d'un couplage } \\
& \text { de } Q \grave{a} Z \text { dans } G\left(\Sigma_{q z \lambda}\right) \text {, et le nombre minimal de sommets de } X \cup Z \\
&\text { dans les deux couplages est le même }\}
\end{aligned}
$$

Nous avons alors $\mathcal{N}_{F}^{*}=\left\{e_{j}\right.$ pour j tel que $\left.x_{j} \in X / \mathcal{J}^{*}\right\}$, où $\mathcal{N}_{F}^{*}$ est le plus petit sousespace fixe contenant les $\mathcal{N}_{\lambda}^{*}$ quand $\lambda$ varie dans $\mathbb{R}^{k}$. En fait le complémentaire de $\mathcal{J}^{*}$ contient toute l'information pertinente sur la perturbation et c'est donc dans cet ensemble que se feront les mesures utiles. On a la série d'inclusions d'espaces suivante :

$$
\mathcal{E} \subset \mathcal{N}_{\lambda}^{*} \subset \mathcal{N}_{F}^{*} \subset \mathcal{V}_{F}^{*} \subset \mathcal{V}_{\lambda}^{*} \subset \operatorname{Ker} C
$$

illustrée sur la Figure 7 dans laquelle les espaces associés à $\mathcal{I}^{*}$ et $\mathcal{J}^{*}$ sont visualisés avec des hachures.

Finalement la condition géométrique (6) peut se traduire pour les systèmes structurés comme suit $[13,12]$. 


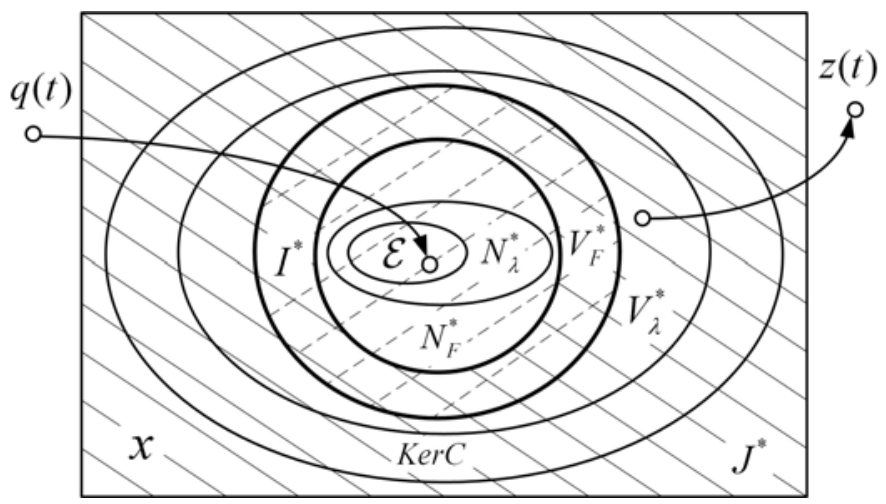

Figure 7. Les inclusions d'espaces

Théorème 4 Considérons un système structuré du type (13) et de graphe $G\left(\Sigma_{q z \lambda}\right)$. Le rejet de perturbation avec retour de mesure est génériquement soluble si et seulement si

$$
\mathcal{I}^{*} \cup \mathcal{J}^{*}=X
$$

Cette condition exprime que le nombre de mesures doit être suffisant et que le temps pour mesurer puis commander doit être inférieur au temps mis par la perturbation pour atteindre la sortie.

\subsection{Exemple des cinq bacs}

Considérons le procédé thermo-hydraulique représenté Figure 8. Ce procédé est composé de 5 bacs, chacun étant alimenté par un débit fixe $F_{1}$ pour les bacs 1 et $3, F_{2}$ pour les bacs 2 et 4 et $\left(F_{1}+F_{2}\right)$ pour le cinquième. L'entrée de commande est la puissance de chauffe $w$ du bac 3 . La sortie à réguler est la température $T_{5}$ du cinquième bac. La perturbation est la température d'alimentation $T_{0}$ et la sortie mesurée $z$ est la température $T_{2}$.

Le problème de rejet de perturbation consiste à trouver une loi de commande par retour de mesure (utilisant uniquement $T_{2}$ ) pour que $T_{5}$ soit insensible aux variations de $T_{0}$. En choisissant comme variables d'état les variations de température autour d'un point de fonctionnement on aboutit à un modèle linéarisé du type (9) où

$$
x=\left[\begin{array}{lllll}
T_{1} & T_{2} & T_{3} & T_{4} & T_{5}
\end{array}\right]^{T}
$$




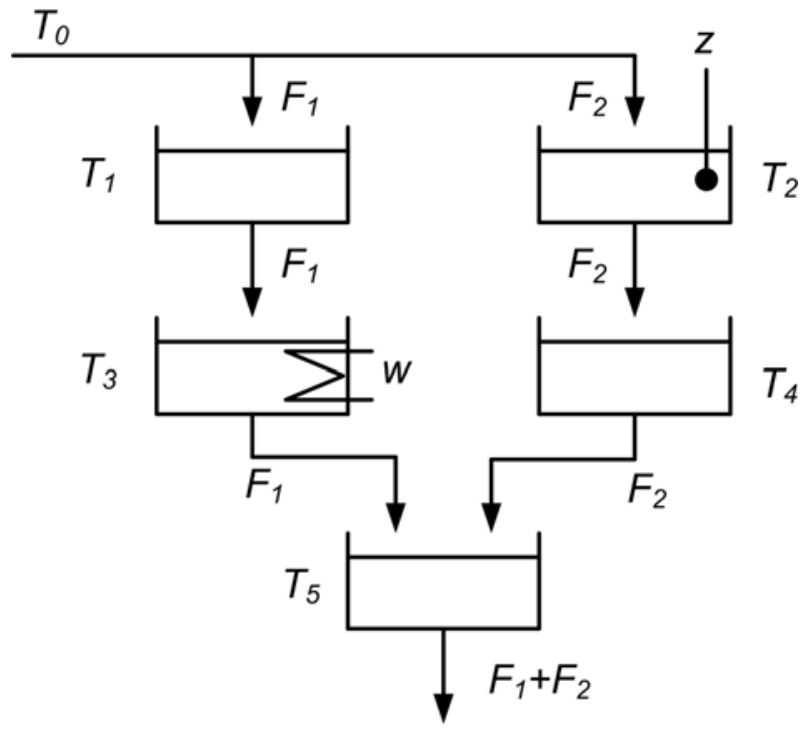

Figure 8. Le procédé à 5 bacs

$q=T_{0}, u=w$ and $y=T_{5}$

avec les matrices suivantes

$$
\begin{gathered}
A=\left[\begin{array}{ccccc}
\frac{-F_{1}}{C_{1}} & 0 & 0 & 0 & 0 \\
0 & \frac{-F_{2}}{C_{2}} & 0 & 0 & 0 \\
\frac{F_{1}}{C_{3}} & 0 & \frac{-F_{1}}{C_{3}} & 0 & 0 \\
0 & \frac{F_{2}}{C_{4}} & 0 & \frac{-F_{2}}{C_{4}} & 0 \\
0 & 0 & \frac{F_{1}}{C_{5}} & \frac{F_{2}}{C_{5}} & \frac{-F_{1}-F_{2}}{C_{5}}
\end{array}\right] \\
B=\left[\begin{array}{c}
0 \\
0 \\
1 / C_{3} \\
0 \\
0
\end{array}\right] ; E=\left[\begin{array}{c}
F_{1} / C_{1} \\
F_{2} / C_{2} \\
0 \\
0 \\
0
\end{array}\right] H=\left[\begin{array}{llllll}
0 & 0 \\
0 & 1 & 0 & 0 & 0
\end{array}\right]
\end{gathered}
$$

$C_{i}$ correspond à la capacité calorifique du bac $i$. A ce modèle on peut aisément associer un système structuré du type (13) dont les matrices sont :

$$
\begin{gathered}
A=\left[\begin{array}{ccccc}
\lambda_{1} & 0 & 0 & 0 & 0 \\
0 & \lambda_{2} & 0 & 0 & 0 \\
\lambda_{3} & 0 & \lambda_{4} & 0 & 0 \\
0 & \lambda_{5} & 0 & \lambda_{6} & 0 \\
0 & 0 & \lambda_{7} & \lambda_{8} & \lambda_{9}
\end{array}\right], B=\left[\begin{array}{c}
0 \\
0 \\
\lambda_{10} \\
0 \\
0
\end{array}\right] ; \\
{\left[\begin{array}{c}
\lambda_{11} \\
\lambda_{12} \\
0 \\
0 \\
0
\end{array}\right] C=\left[\begin{array}{lllll}
0 & 0 & 0 & 0 & \lambda_{13}
\end{array}\right]} \\
H=\left[\begin{array}{lllll}
0 & \lambda_{14} & 0 & 0 & 0
\end{array}\right]
\end{gathered}
$$


Le graphe associé est donné Figure 9 : En utilisant l'algorithme itératif donné dans [9]

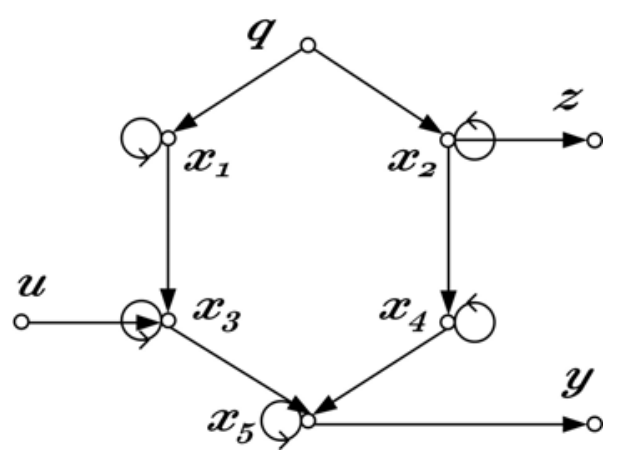

Figure 9. Graphe de l'exemple des bacs

$$
\left\{\begin{array}{l}
V_{0}=\operatorname{KerC} \\
V_{k}=\operatorname{Ker} C \cap A^{-1}\left(V_{k-1}+\operatorname{Im} B\right)
\end{array}\right.
$$

on peut calculer le sous-espace $(A, B)$-invariant maximum contenu dans $\operatorname{Ker} C, \mathcal{V}_{\lambda}^{*}$.

$$
\mathcal{V}_{\lambda}^{*}=\operatorname{Span}\left[\begin{array}{ccc}
1 & 0 & 0 \\
0 & 1 & 0 \\
0 & 0 & 1 \\
0 & 0 & -\lambda_{7} / \lambda_{8} \\
0 & 0 & 0
\end{array}\right]
$$

Le sous-espace, partie fixe de $\mathcal{V}_{\lambda}^{*}$ et noté $\mathcal{V}^{*}{ }_{F}$ est clairement

$$
\mathcal{V}_{F}^{*}=\operatorname{Span}\left[\begin{array}{ll}
1 & 0 \\
0 & 1 \\
0 & 0 \\
0 & 0 \\
0 & 0
\end{array}\right]
$$

De façon duale calculons $\mathcal{N}^{*}$ le plus petit sous-espace $(H, A)$-invariant contenant Im $E$ en utilisant l'algorithme itératif

$$
\left\{\begin{array}{l}
N_{0}=I m E \\
N_{k}=A\left(N_{k-1} \cap K e r H\right)+\operatorname{ImE}
\end{array}\right.
$$

pour obtenir finalement

$$
\mathcal{N}_{\lambda}^{*}=\operatorname{Span}\left[\begin{array}{c}
\lambda_{11} \\
\lambda_{12} \\
0 \\
0 \\
0
\end{array}\right]
$$


Sa partie fixe est donnée par $\mathcal{N}_{F}^{*}$

$$
\mathcal{N}_{F}^{*}=\operatorname{Span}\left[\begin{array}{cc}
1 & 0 \\
0 & 1 \\
0 & 0 \\
0 & 0 \\
0 & 0
\end{array}\right]
$$

Pour presque toute valeur des paramètres la condition (12) est satisfaite, à savoir

$$
\mathcal{E} \subset \mathcal{N}_{\lambda}^{*} \subset \mathcal{N}_{F}^{*} \subset \mathcal{V}_{F}^{*} \subset \mathcal{V}_{\lambda}^{*} \subset \operatorname{Ker} C
$$

Le rejet de perturbation avec retour de mesure est donc génériquement soluble.

Par l'approche graphique on peut calculer les ensembles de sommets $\mathcal{I}^{*}$ et $\mathcal{J}^{*}$ qui valent respectivement

$$
\mathcal{I}^{*}=\left\{x_{1}, x_{2}\right\}, \mathcal{J}^{*}=\left\{x_{3}, x_{4}, x_{5}\right\}
$$

Donc $\mathcal{I}^{*} \cup \mathcal{J}^{*}=X$ et le problème a une solution par le Théorème 4 .

Dans la pratique, la mesure de la température du deuxième bac est une information suffisamment rapide sur la perturbation $T_{0}$ pour qu'elle soit prise en compte par l'action de $w$ dans le bac 3 et que la perturbation $T_{0}$ soit ensuite compensée à temps au niveau du bac 5.

Un point intéressant que nous n'avons pas considéré est celui de la stabilité du système en boucle fermée avec la loi de commande par retour de mesure. En général la stabilité dépend de la valeur des paramètres du modèle. Dans le cas de notre exemple, en utilisant les travaux géométriques de [15] on peut analyser la stabilité en boucle fermée. On peut montrer que pour tout choix de paramètres correspondant à un modèle physique (volumes des bacs, débits, capacités calorifiques positifs) le système en boucle fermée sera stable et rejettera bien les perturbations.

\section{Conclusion}

Dans ce papier on a survolé l'approche structurelle des systèmes en se focalisant sur un problème classique mais non trivial, le rejet de perturbations par retour de mesure. On a illustré sur un exemple simple de système physique hydraulique que la connaissance de la seule structure interne du système permettait de dire si le problème était soluble génériquement indépendamment de la valeur des paramètres. Avec le même type d'approche il est possible de résoudre d'autres problèmes de commande, d'observation et de diagnostic. Pour le diagnostic, on s'est en particulier intéressés au problème de localisation de capteurs pour détecter et identifier des défauts [16]. Notre intérêt actuel se porte sur la classification de capteurs potentiellement défaillants en fonction de leur criticité relativement à la résolution de divers problèmes comme l'observation [17], le rejet de perturbations et le diagnostic [18].

\section{Remerciements}

Les auteurs remercient Do Hieu Trinh pour sa participation à la présentation de cette communication. 


\section{Bibliographie}

[1] C. Lin, "Structural controllability," IEEE Trans. Automat. Control, vol. 19, pp. 201-208, 1974.

[2] M. Blanke, M. Kinnaert, J. Lunze, and M. Staroswiecki, Diagnosis and Fault-tolerant Control. Springer-Verlag, 2003.

[3] K. Reinschke, Multivariable Control, A Graph-theoretic Approach. Springer-Verlag, 1988.

[4] K. Murota, Matrices and Matroids for Systems Analysis, ser. Algorithms and Combinatorics 20. Springer, 2000.

[5] J. M. Dion, C. Commault, and J. van der Woude, "Generic properties and control of linear structured systems : a survey," Automatica, vol. 39, no. 7, pp. 1125-1144, 2003.

[6] M. Bahar, J. Jantzen, C. Commault, J. M. Dion, and V. Hovelaque, "Structural decoupling and disturbance rejection in a distillation column," in World Congress IFAC'96, vol. M, San Francisco, USA, 1996.

[7] J. van der Woude, "A graph theoretic characterization for the rank of the transfer matrix of a structured system,” Math. Control Signals Systems, vol. 4, pp. 33-40, 1991.

[8] G. Basile and G. Marro, Controlled and Conditioned Invariants in Linear System Theory. Prentice Hall, 1992.

[9] W. Wonham, Linear multivariable control : a geometric approach. Springer Verlag, 1979.

[10] C. Commault and J. M. Dion, "A comprehensive introduction to the geometric theory of linear multivariable systems," IEEE Trans. Educ., vol. 35, no. 1, pp. 92-97, 1992.

[11] C. Commault, J. M. Dion, and A. Perez, "Disturbance rejection for structured systems," IEEE Trans. Automat. Control, vol. 36, pp. 884-887, 1991.

[12] J. van der Woude, "Disturbance decoupling by measurement feedback for structured systems : a graph theoretic approach," in Proc.2-th European Control Conference ECC'93, Groningen, 1993, pp. 1132-1137.

[13] C. Commault, J. M. Dion, and V. Hovelaque, "A geometric approach for structured systems : Application to the disturbance decoupling problem," Automatica, vol. 33, pp. 403-409, 1997.

[14] J. Schumacher, "Compensator synthesis using (C,A,B) pairs," IEEE Trans. Automat. Control, vol. AC-25, pp. 1133-1138, 1980.

[15] J. Willems and C. Commault, "Disturbance decoupling by measurement feedback with stability or pole placement," SIAM J. Cont. and Opt., vol. 19, pp. 490-504, 1981.

[16] C. Commault and J. Dion, "Sensor location for diagnosis in linear systems : a structural analysis," IEEE Trans. Autom. Control, vol. 52, no. 2, pp. 155-169, 2007.

[17] C. Commault, J. Dion, and D. Trinh, “Observability preservation under sensor failure,” IEEE Trans. Autom. Control, à paraître, 2008.

[18] C. Commault, J. Dion, and S. Yacoub, "Sensor classification for the fault detection and isolation problem,” in IFAC Symp. SSC 07, Iguazu Falls, Brazil, 2007. 